\title{
A Radio Resource Allocation Scheme for Fixed Broadband Wireless Access Systems with Avoidance of Major Interferers
}

\author{
N. VAIOPOULOS, A. VAVOULAS, D. VAROUTAS and T. SPHICOPOULOS \\ Department of Informatics and Telecommunications, University of Athens, Greece \\ E-mails:nvaio@di.uoa.gr,vavoulas@di.uoa.gr,arkas@di.uoa.gr,thomas@di.uoa.gr
}

\begin{abstract}
An improved radio resource allocation scheme with avoidance of major interferers is proposed and analyzed for the downlink of Fixed Broadband Wireless Access (FBWA) systems with full frequency reuse. The scheme is based on Enhanced Staggered Resource Allocation (ESRA) and permits the enhancement of the throughput per sector. Simulation results show a maximum downlink throughput per sector in excess of $44 \%$ and an increase of $10 \%$ with respect to ESRA is achieved, with Base Station (BS) selection procedure, while meeting a $15 \mathrm{~dB}$ signal-to-interference ratio (SIR).
\end{abstract}

Keywords: cochannel interference, channel allocation, interference suppression, radio resource management

\section{Introduction}

As the demand for integrated broadband services (e.g. interactive television, tele/video conferencing, etc.) increases, further investments from the network operators are needed. Since the deployment of fiber solutions leads to higher investment risks [1], different technology alternatives able to support broadband access appear such as fixed broadband wireless access (FBWA). A typical FBWA system comprises of cells which are divided into multiple sectors, in order to increase system capacity and spectral efficiency, and the associated with each cell BS, equipped with sector antennas. Subscribed terminals with rooftop directional antennas are communicating directly with their corresponding BS, which might be connected to a backbone network. But, as the number of subscribed terminals per cell increase, the licensed spectrum must be reused and therefore co-channel interference arises and becomes the major problem to solve and a critical factor responsible for performance degradation.

Co-channel interference is caused by undesirable concurrent transmissions (i.e. multiple sector antennas that point directly to a terminal antenna). The mechanism of these transmissions is separate for intercell and intracell interference. Intercell interference arises from concurrent transmissions from terminals (uplink direction) or BS (downlink direction) in all cells except the cell of interest while intracell interference is generated from concurrent transmissions within the serving cell. It is the primary goal of resource assignment techniques to schedule appropriately these transmissions so as to mitigate the total interference and to maximize the total throughput and coverage.

Several resource assignment methods have been proposed for the downlink of FWBA systems [2-5]. In this paper, a radio resource allocation method based on the Enhanced Staggered 
Resource Allocation (ESRA) method [4] is proposed and analyzed, for the downlink segment of FWBA, leading to an improvement of the throughput per sector for increased traffic load conditions. The basic idea of the proposed algorithm is the avoidance of the major interferers by using different allocation schemes for the even and odd sectors. The rest of paper is organized as follows. In Sections 2, a short description of the ESRA method is given whereas the details of the proposed resource allocation algorithm and the downlink performance are described in Section 3. Section 4 illustrates and discusses simulation results whereas concluding remarks are given in Section 5.

\section{The Enhanced Staggered Resource Allocation (ESRA) Method}

As it has been proposed in [4], ESRA examines a packet switched broadband wireless network using time division multiple access (TDMA) technique and time division duplexing (TDD) with full frequency reuse. The service area is divided in hexagonal cells and sectors are labeled from 1 to 6 , counter-clockwise, in such a way that there are no adjacent sectors bearing the same label (Figure 1). The frame is divided into six subframes, which are further divided into mini-frames labeled from 1 to 6.

Each sector schedules packets for transmissions in available mini-frames of each subframe following the staggered order of Figure 2. According to this order, sector 1 schedules packets for transmissions in subframe 1 (denoted by a). For further packet transmissions, exploiting BS directional antennas, it uses subframe 4, which is the first subframe of the opposite sector. The next options for sector 1 will be the available mini-frames of the first subframes of the other two opposite sectors (i.e. sectors 5 and 3) clockwise and the last two options will be the available mini-frames of the first subframes of the adjacent intracell sectors (i.e. sectors 2 and 6) clockwise too. The same procedure is applied to the other sectors.

Terminals are classified on six classes according to the number of maximum tolerable concurrent transmissions following the staggered order of Figure 2. For example, a class 3

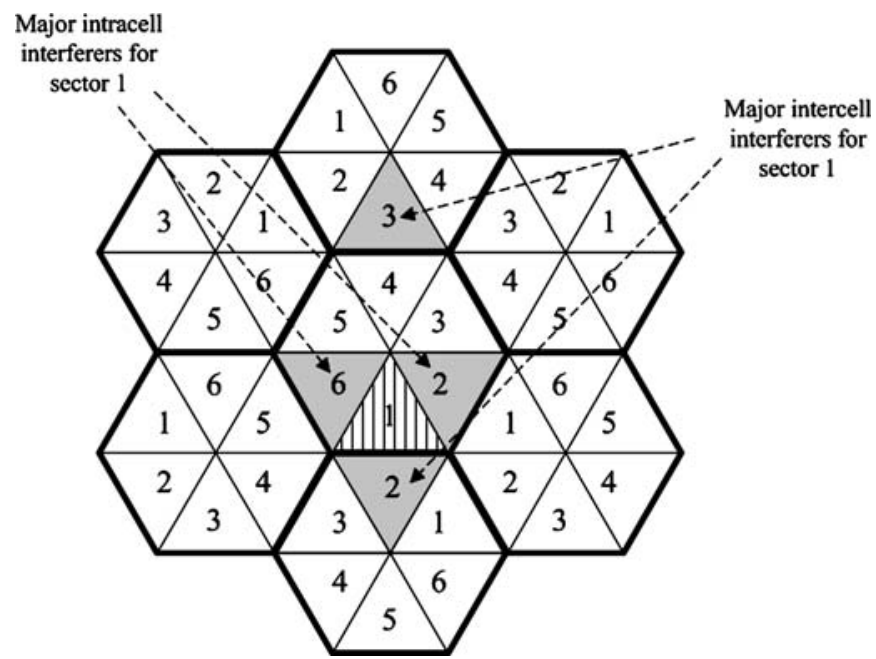

Figure 1. Major interferers for the downlink direction of the hexagonal cell layout. 


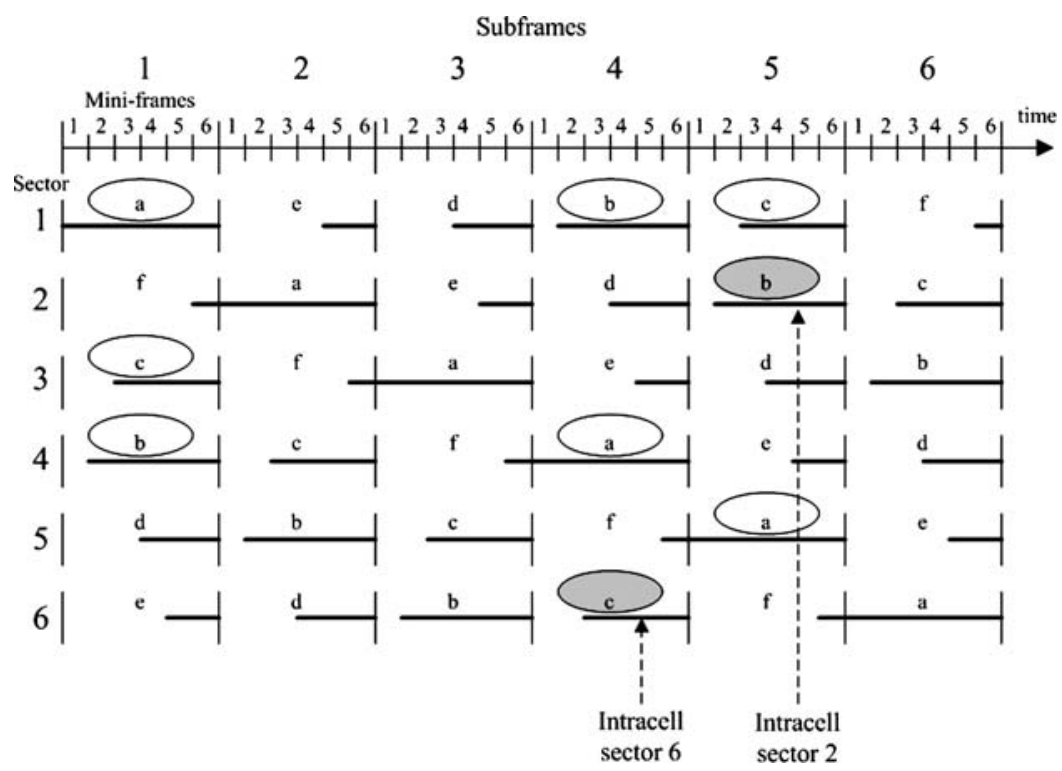

Figure 2. Three concurrent transmissions for sector 1 according to ESRA (two dominant intracell interferers).

ESRA class 3 terminals

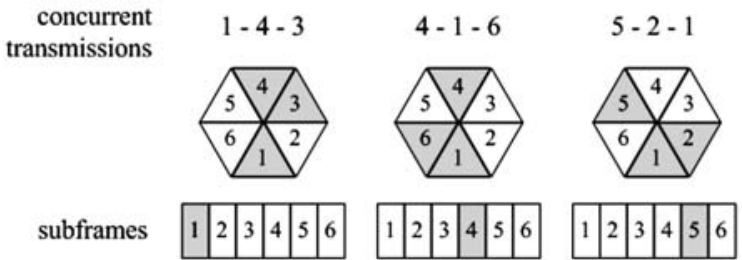

ESRA class 4 terminals

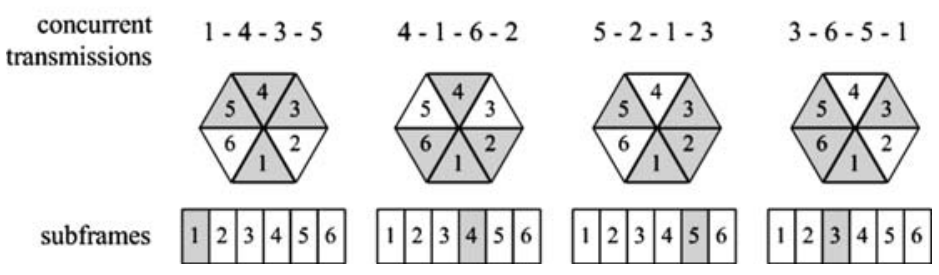

Figure 3. Class 3 and class 4 terminals according to ESRA method.

terminal of sector 1 tolerates the following three concurrent transmissions: sectors 1-4-3 in subframe 1, sectors 4-1-6 in subframe 4 and sectors 5-2-1 in subframe 5 (Figure 3). The degree of concurrent transmissions of each mini-frame is defined on its label. More precisely, mini-frame 1 allows only 1 packet transmission; mini-frame 2 allows 2 concurrent packet transmissions and so on. Terminal classification is based on the reception quality of their location that depends on antenna characteristics (3dB beamwidth and Front-To-Back (FTB) ratio), shadowing and distance from serving BS. The reception quality may be improved through a macrodiversity procedure (i.e. selection of serving sector). Each mini-frame with the same label has the same number of timeslots in each subframe (mini-frame $i$ has $n_{i}, i=1$, 
$2 \ldots 6$ timeslots in each subframe). The size of mini-frames is chosen to match the expected traffic load and is a mechanism to increase the overall throughput.

\section{Resource Allocation with Avoidance of Major Interferers (RA-AMI)}

For a typical FBWA system, throughput enhancement is achieved by upgrading the number of higher classes terminals through advanced methods of major intercell and intracell interferers avoidance. As shown in Figure 1, the major interferers for sector 1, under a simple path loss model, comes from the shadowed intracell sectors 2 and 6 (due to overlapping sector antenna patterns) as well as from shadowed intercell sector 3 (because of the front lobe of sector antenna 3 that point directly to the terminals of the tagged sector) and the opposite shadowed sector 2. Following the ESRA staggered order (Figure 2) for higher terminal classes (i.e. classes 4,5 and 6) the major intracell interferers are appearing not only solely but also together. In the case of class 4 terminals, as shown in Figure 3, sector 6 interferes sector's 1 terminals in combination 3-6-5-1, sector 2 in combination 5-2-1-3 and both sectors 6 and 2 in combination 4-1-6-2. So, the motivation for this work is to further examine the throughput enhancement possibilities through the increase of the lower class terminals.

The ESRA framework is adopted as far as terminal classification, mini-frame structure, sector selection procedure and scheduling mechanism for packet transmissions are concerned. The maximum downlink throughput per sector for the system is given by [4]:

$$
\gamma=\sum_{i} \frac{i n_{i}}{K N}
$$

where $K$ is the number of sectors in each cell and $n_{i}$ the number of timeslots per mini-frame i:

$$
n_{i}=\left[\frac{N_{t} a_{i} / i}{\sum_{j} a_{j} / j}\right]
$$

where $a_{i}$ is the percentage of class $i$ terminals, $N_{t} \approx N$ the number of timeslot in each subframe and $[x]$ the closest integer to $x$. So, it is clear from (1), (2) that the maximum throughput enhancement is relevant to the fractions of terminals in the defined classes and is achieved by upgrading a portion of terminals from a lower class to an upper class.

As shown in Figure 3, following the ESRA order for sector 1, class 3 terminals must tolerate both major intracell interferers (i.e. sector 6 in combination 4-1-6 and sector 2 in combination 5-2-1) resulting thus in degradation of class 3 terminals.

The RA-AMI method is based on a different allocation scheme for odd and even sectors, which is examined and proposed as follows.

The sector 1 schedules packets for transmission in subframe 1, as shown in Figure 4. If there are more packets for transmission, it uses the available mini-frames of subframe 4 , which are the first subframe of the opposite sector, in order to exploit the BS directional antennas and the low level of interference. All sectors follow this procedure for the first two subframes.

However, the next two options for sector 1, according to the staggered order will be the available mini-frames of the first subframes of the other two opposite sectors (i.e. sectors 5 and 3 ) clockwise and the last two options will be the available mini-frames of the first subframes of the two adjacent intracell sectors (i.e. sectors 6 and 2) counter-clockwise. This concept is applied to the odd labeled sectors (i.e. sectors 3 and 5). 


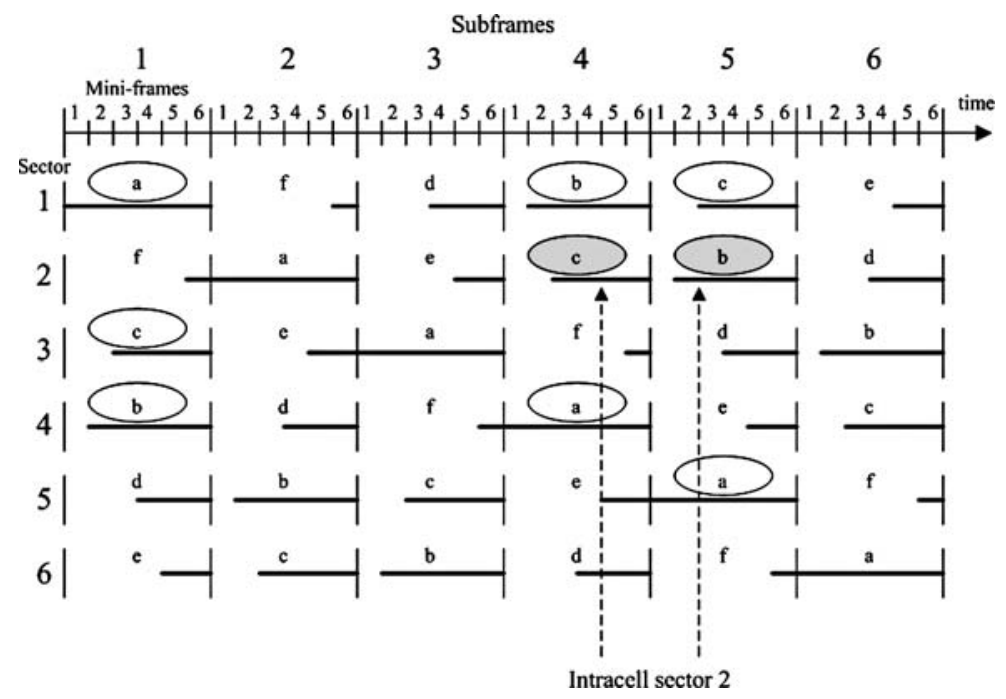

Figure 4. Three concurrent transmissions for sector 1 according to RA-AMI (one dominant intracell interferer).

On the contrary, for sector 2 , the next two options will be the available mini-frames of the first subframes of the other two opposite sectors (i.e. sectors 4 and 6) counter-clockwise while the last two resorts will be the available mini-frames of the first subframes of the two adjacent intracell sectors (i.e. sectors 3 and 1) clockwise. This procedure is repeated for the even labeled sectors (i.e. sectors 4 and 6).

This situation is exploited by the RA-AMI method in order to increase the number of class 3 terminals due to the fact that each sector is interfered only by one adjacent intracell sector in contrast with ESRA where each sector is interfered by both adjacent intracell sectors one after the other.

More explicitly, in the case of three concurrent transmissions (class 3 terminals) of sector 1, Figure 2 presents the allocation scheme based on ESRA and Figure 4 the proposed scheme. Sector 1, following the ESRA staggered order, is interfered in subframe 4 by sector 6 (one dominant intracell interferer) and in subframe 5 by sector 2 (the other dominant intracell interferer). On the other hand, following the RA-AMI staggered order, sector 1 is interfered in subframes 4 and 5 by sector 2 (i.e. the same dominant intracell interferer). As a result, the proposed method upgrades the fraction of terminals that tolerate three concurrent transmissions enhancing the maximum throughput per sector according to (1).

\section{Results and Discussion}

Simulation results have been obtained in order to study the terminal classification and the maximum throughput (packets/slot/sector) of ESRA and RA-AMI methods. A two-tier cell layout consisting of seven hexagonal cells with six sectors each have been considered. To avoid border effects only the statistics in the middle cell are presented here, while 1000 terminals are randomly placed over sector 1 . Typical patterns are used for BS and terminal antennas with FTB ratio of $25 \mathrm{~dB}$ and $15 \mathrm{~dB}$ respectively, while a $3 \mathrm{~dB}$ beamwidth of 50 and 30 degrees is incorporated correspondingly. A simple path loss model, with an exponent of 4 and lognormal shadowing with zero mean and standard deviation equal to 8 , is adopted. The reference SIR threshold for success packet transmission is taken to be equal to $15 \mathrm{~dB}$. 
484 N. Vaiopoulos et al.

Table 1. Fraction of terminals to various classes for ESRA and RA-AMI

\begin{tabular}{lrrrrr}
\hline \multirow{2}{*}{ Terminal class } & \multicolumn{2}{c}{ Without BS selection } & & \multicolumn{2}{c}{ With BS selection } \\
\cline { 2 - 3 } \cline { 5 - 6 } & \multicolumn{1}{c}{ ESRA } & RA-AMI & & ESRA & RA-AMI \\
\hline 1 & $7.60 \%$ & $7.60 \%$ & & $4.15 \%$ & $4.14 \%$ \\
2 & $55.36 \%$ & $34.47 \%$ & & $59.15 \%$ & $34.60 \%$ \\
3 & $3.61 \%$ & $24.50 \%$ & & $4.26 \%$ & $28.89 \%$ \\
4 & $2.85 \%$ & $2.85 \%$ & & $3.37 \%$ & $3.36 \%$ \\
5 & $3.80 \%$ & $3.80 \%$ & & $4.49 \%$ & $4.48 \%$ \\
6 & $17.38 \%$ & $17.38 \%$ & & $20.54 \%$ & $20.49 \%$ \\
Coverage & $90.60 \%$ & $90.60 \%$ & & $95.96 \%$ & $95.96 \%$ \\
Throughput & $36.96 \%$ & $40.41 \%$ & $39.68 \%$ & $44.15 \%$ \\
\hline
\end{tabular}

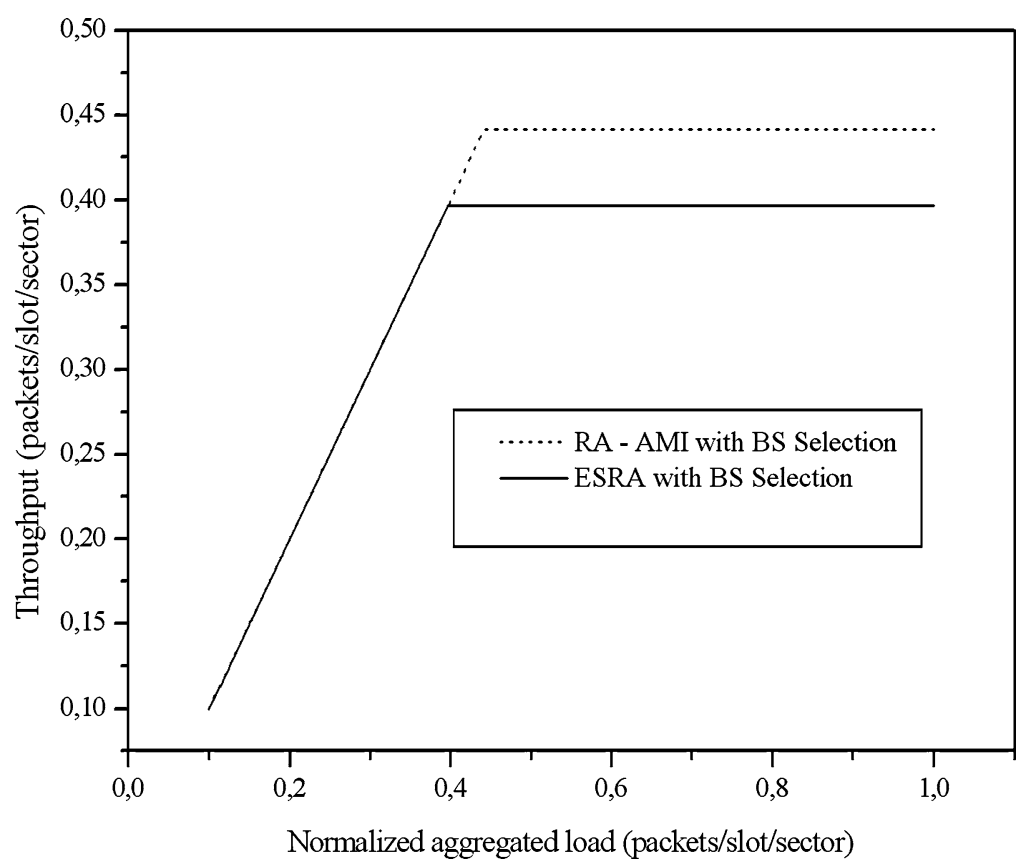

Figure 5. Downlink comparison of the ESRA and RA-AMI methods with BS Selection.

Following the ESRA order [4], Table 1 summarizes the fraction of terminals into 6 classes for both methods, with and without BS selection and sector selection. The sum of the fractions for all classes gives the total percentage of terminals that can be served by both methods and is mentioned as coverage. The coverage for both methods is the same since it is determined by intercell interference, which is avoided in the same manner in both methods. Without BS selection, the coverage is $90.60 \%$, as shown in Table 1, while in the case of BS selection it is $95.96 \%$. It becomes clear from Table 1 that the difference between ESRA and RA-AMI methods for class 3 terminals is more than $20 \%$ either with or without BS selection. As a result, an increment of $3.45 \%$ and $4.47 \%$ of maximum throughput per sector is achieved with and without BS selection correspondingly. This happens because with RA-AMI more terminals can tolerate more concurrent transmissions. 
Figure 5 compares the throughput of ESRA and RA-AMI methods as a function of normalized aggregated load using BS selection. For a normalized aggregated load up to $39.68 \%$ both methods achieve the same throughput. For normalized aggregated load between $39.68 \%$ and $44.15 \%$ ESRA is maintained at $39.68 \%$ while RA-AMI is further increased until $44.15 \%$ is reached. As traffic increases more, the difference between the two methods remains fixed at $4.47 \%$, which is the difference between the maximum throughputs of both methods. The same comments apply without using BS selection, where the difference between the two methods remains fixed at $3.45 \%$ for value above $40.41 \%$ of normalized aggregated load.

\section{Conclusion}

A radio resource allocation method for the downlink direction of a fixed broadband wireless access system is proposed and analyzed. This approach is based on ESRA method thus enhancing the throughput per sector by increasing the number of terminals that tolerate more concurrent transmissions. For a typical radio environment a maximum throughput per sector close to $44 \%$ and an upgrading with respect to ESRA method of 4,47 percentage units (10\% improvement) are achieved with the RA-AMI method with BS Selection, maintaining the packet success probability equal to one.

\section{References}

1. T. Monath, N.K. Elnegaard, P. Cadro, D. Katsianis, and D. Varoutas, "Economics of Fixed Broadband Access Network Strategies”, IEEE Commun. Mag., Vol. 41, pp. 132-139, 2003.

2. K. Chawla and X.X. Qiu, "Quasi-Static Resource Allocation with Interference Avoidance for Fixed Wireless Systems", IEEE Journal on Selected Areas in Communications, Vol. 17, pp. 493-504, 1999.

3. T.K. Fong, P.S. Henry, K.K. Leung, X.X. Qiu, and N.K. Shankaranarayanan, "Radio Resource Allocation in Fixed Broadband Wireless Networks", IEEE Transactions on Communications, Vol. 46, pp. 806-818, 1998.

4. K.K. Leung and A. Srivastava, "Dynamic Allocation of Downlink and Uplink Resource for Broadband Services in Fixed Wireless Networks ", IEEE Journal on Selected Areas in Communications, Vol. 17, pp. 9901006, 1999.

5. V. Tralli, R. Veronesi, and M. Zorzi, "Power-Shaped Advanced Resource Assignment (PSARA) for Fixed Broadband Wireless Access Systems ", IEEE Transactions on Wireless Communications, Vol. 3, pp. 22072220, 2004.

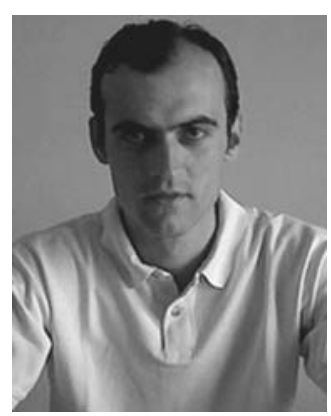

Nicholas Vaiopoulos was born in Lamia in 1977. He received his Physics degree and his M.Sc. degree in electronics and radio-communications from the University of Athens, Greece in 2000 
and 2003, respectively. Currently, he is working towards his Ph.D. degree on the resource allocation techniques with reference to wireless systems at the Department of informatics and Telecommunications at the same University. His research interests include broadband communications systems, scheduling algorithms and power control techniques for wireless systems.

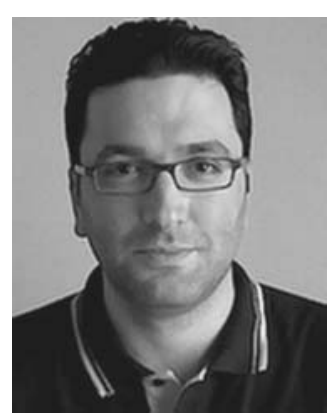

Alexander Vavoulas was born in Athens in 1976. He received his B.Sc. degree in physics and the M.Sc. degree in electronics and radio-communications in 2000 and 2002 respectively, both from the University of Athens, Greece. Currently he is working toward the Ph.D. degree on the radio resource allocation techniques with the same University. His research interest is focused on broadband wireless access and interference management. He is a student nmember of the IEEE.

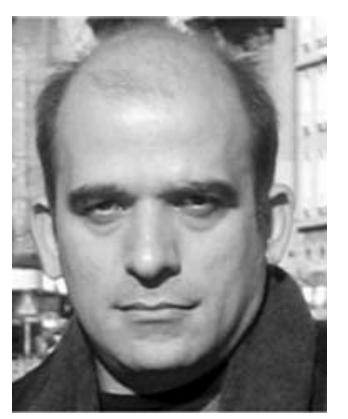

Dimitris Varoutas holds BSc. degree in Physics, M.Sc. in electronics and radio-communi cations and Ph.D. in telecommunications systems and technoeconomics, all from the University of Athens. He is a lecturer in the Department of Informatics and Telecommunications of University of Athens and an adjunct assistant professor in the Department of Telecommunications of the newly founded University of Peloponnese. He has participating in numerous European R\&D projects in the RACE I \&II, ACTS, Telematics, RISI and IST framework in the areas of telecommunications and Technoeconomics. He is an adviser in several organisations including OTE and EETT (Greek NRA for telecommunications) in the fields of telecommunications, broadband and mobile services, licensing, spectrum management, pricing and legislation. His research interests are optical, microwave communications and technoeconomic evaluation of network architectures and services. He has more than 30 publications in refereed journal and conferences in the area of telecommunications, optoelectronics and technoeconomics. He is a member of IEEE and serves as reviewer in several journals and conferences. 


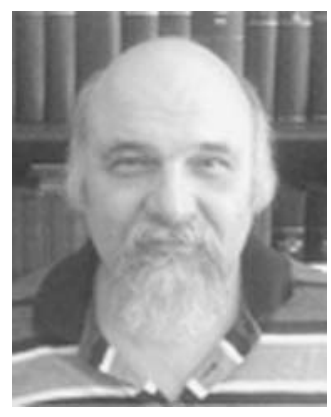

Thomas Sphicopoulos received the Physics degree from Athens University in 1976, the D.E.A. degree and Doctorate in Electronics both from the University of Paris VI in 1977 and 1980 respectively, the Doctorat Es Science from the Ecole Polytechnique Federale de Lausanne in 1986. From 1976 to 1977 he worked in Thomson CSF Central Research Laboratories on Microwave Oscillators. From 1977 to 1980 he was an Associate Researcher in Thomson CSF Aeronautics Infrastructure Division. In 1980 he joined the Electromagnetism Laboratory of the Ecole Polytechnique Federal de Lausanne where he carried out research on Applied Electromagnetism. Since 1987 he is with the Athens University engaged in research on Broadband Communications Systems. In 1990 he was elected as an Assistant Professor of Communications in the Department of Informatics \& Telecommunications, in 1993 as Associate Professor and since 1998 he is a Professor in the same Department. His main scientific interests are Microwave and Optical Communication Systems and Networks and Technoeconomics. He has lead about 40 National and European R\&D projects. He has more than 100 publications in scientific journals and conference proceedings. From 1999 he is advisor in several organisations including EETT (Greek NRA for telecommunications) in the fields of market liberalisation, spectrum management techniques and technology convergence. 